\title{
Exposure to ambient fine particulate matter is associated with changes in fasting glucose and lipid profiles: a nationwide cohort study
}

Woo-young Shin ${ }^{1 *}$, Jung-ha Kim¹', Gyeongsil Lee ${ }^{2}$, Seulggie Choi ${ }^{3}$, Seong Rae Kim ${ }^{4}$, Yun-Chul Hong ${ }^{5,6,7}$ and Sang Min Park ${ }^{2,3^{*}}$

\begin{abstract}
Background: Ambient fine particulate matter is a rising concern for global public health. It was recently suggested that exposure to fine particulate matter may contribute to the development of diabetes and dyslipidaemia. This study aims to examine the potential associations of ambient particulate matter exposure with changes in fasting glucose and lipid profiles in Koreans.

Method: We used the data from the National Health Insurance Service-National Sample Cohort (NHIS-NSC), a nationwide database representative of the Korean population. A total of 85,869 individuals aged $\geq 20$ years were included. Multiple regression analyses were conducted to assess the associations between exposure to particulate matter and changes in fasting glucose and lipid profiles at 2-year intervals after adjusting for confounders.

Results: Significant associations were observed between an increase in interquartile range for particulate matter $<2.5 \mu \mathrm{m}$ in diameter $\left(\mathrm{PM}_{2.5}\right)$ and elevated levels of fasting glucose and low-density lipoprotein cholesterol $(p$ for trend $=0.015$ and 0.010, respectively), while no association for particulate matter sized 2.5-10 $\mu \mathrm{m}$ in diameter ( $\left.\mathrm{PM}_{10-2.5}\right)$ was noted after adjusting for the other covariates. Sub-group analyses showed stronger associations in individuals who were older ( $\geq 60$ years) or physically inactive.
\end{abstract}

Conclusions: Fine particulate matter exposure affects worsening fasting glucose and low-density lipoprotein cholesterol levels, with no evidence of an association for coarse particulate matter.

Keywords: Air pollution, Particulate matter, Glucose, Lipid, Cohort study

\footnotetext{
*Correspondence: swy84@caumc.or.kr; smpark.snuh@gmail.com

'Department of Family Medicine, Chung-ang University Medical Center,

Seoul 06973, Republic of Korea

${ }^{2}$ Department of Family Medicine and Biomedical Sciences, College of

Medicine, Seoul National University, 101 Daehak-ro, Jongno-gu, Seoul 03080,

Republic of Korea

Full list of author information is available at the end of the article
}

(c) The Author(s). 2020 Open Access This article is licensed under a Creative Commons Attribution 4.0 International License, which permits use, sharing, adaptation, distribution and reproduction in any medium or format, as long as you give appropriate credit to the original author(s) and the source, provide a link to the Creative Commons licence, and indicate if changes were made. The images or other third party material in this article are included in the article's Creative Commons licence, unless indicated otherwise in a credit line to the material. If material is not included in the article's Creative Commons licence and your intended use is not permitted by statutory regulation or exceeds the permitted use, you will need to obtain permission directly from the copyright holder. To view a copy of this licence, visit http://creativecommons.org/licenses/by/4.0/. The Creative Commons Public Domain Dedication waiver (http://creativecommons.org/publicdomain/zero/1.0/) applies to the data made available in this article, unless otherwise stated in a credit line to the data. 


\section{Précis}

Fine particulate matter exposure affects to worsen fasting glucose and low-density lipoprotein cholesterol levels, with stronger associations in individuals who were older or physically inactive.

\section{Background}

Cardiovascular disease and diabetes have become the major causes of mortality and public health concerns around the world. It is estimated that approximately 20 million people die of these diseases annually [1]. Similarly, these diseases accounted for approximately 24.3\% of the causes of death among the Korean population in 2016, and the rates of morbidity and mortality related to these diseases are continuously increasing [2,3]. Accordingly, there have been various studies and approaches to identify or manage them in Korea, as well as worldwide.

Several countries, including South Korea, that experienced industrialization and urbanization in recent decades have aggravated atmospheric quality and air pollution. These conditions have raised concern regarding the leading risk factors for the global burden of diseases [4, 5]. Ambient particulate matter (PM) air pollution accounted for approximately $7.5 \%$ of deaths and was the sixth highest factor contributing to disability-adjusted life years in 2016 [1]. PM refers to a widespread air pollutant consisting of a mixture of variable particles, including sulfates, nitrates, organic materials, particle-bound water, metals, or even biological components suspended in the air [6]. Its ambient exposure is ubiquitous and involuntary, affecting large populations. Several studies on the health effects of PM air pollution have been actively conducted in many countries. PM exposure is well known for its detrimental effects on the cardiovascular and respiratory systems and total mortality [5, 7, 8]. Some epidemiological studies have recently suggested that exposure to ambient PM may contribute to harmful health effects on metabolic systems, such as increased risks of hyperglycaemia and dyslipidaemia $[9,10]$, which are known as wellestablished cardiometabolic risks with strong evidence for developing diabetes and cardiovascular events [11].

However, limited studies have focused on the relationship of PM exposure with hyperglycaemia and dyslipidaemia, with most being conducted as cross-sectional designs for short-term periods among relatively few participants. Furthermore, to the best of our knowledge, few studies have explored the effects of PM concentrations on individual changes in cardiometabolic risks using a large cohort. Therefore, the aim of this study was to examine the associations between the concentrations of PM exposure and changes in levels of blood glucose and lipid profiles among Korean adults using a nationwide cohort.

\section{Methods}

\section{Data source and study population}

The National Health Insurance Service-National Sample Cohort (NHIS-NSC) is a nationwide population-based cohort with a substantial volume of information regarding citizens' health examinations and utilization of health insurance among a representative Korean population [12]. The NHIS-NSC database was constructed for research and policy development by the National Health Insurance Service (NHIS). This study is based on data from the NHIS-NSC obtained from 2002 to 2013, which includes participants' sociodemographic variables and health examination results (Project number: NHIS-2018-2-135).

A total of $1,006,481$ individuals were randomly selected using a systematic stratified sampling method, maintaining approximately $2.2 \%$ of the entire eligible Korean population since 2002, followed-up until 2013 [12]. Of these, 223,491 participants aged $<20$ years old were excluded in this study, considering factors influencing children or adolescents' exposure to PM. Children or adolescents' exposure to environmental contaminants, including PM, is expected to be different from adults due to differences in their physiologic characteristics and behavioural patterns [13]. Therefore, this study was designed and conducted to evaluate the health effects of PM exposure in adults. In addition, we excluded 527,082 participants whose data on $\mathrm{PM}_{10}$ or $\mathrm{PM}_{2.5}$ levels were not available during the study periods, as this study aimed to provide potential evidence of the size-specific effects of PM exposure by evaluating the adverse effects of both fine and coarse PM on changes in laboratory values. Of the remaining 255,908 participants, we excluded 169,125 participants who did not take two biennial health examinations consecutively during 2010 2013. A total of 85,869 (43,595 men and 42,274 women) individuals were finally analysed after excluding participants who had missing data on the results of health examinations and questionnaires.

Informed consent was obtained for the NHIS-NSC from all participants. This study was approved by the institutional review board of Seoul National University (IRB number: E-1803-045-928).

\section{Assessment of exposure to particulate matter}

We obtained atmospheric monitoring data for the daily mean concentrations of hourly measured PM with aerodynamic diameters of $\leq 10 \mu \mathrm{m} \quad\left(\mathrm{PM}_{10}\right)$ and $\leq 2.5 \mu \mathrm{m}$ $\left(\mathrm{PM}_{2.5}\right)$ from the National Ambient Air Monitoring Information System in the Ministry of Environment of Korea [14]. The hourly exposure concentrations were measured using the beta-ray absorption method, following the standard reference protocol from the Korean Air Pollutants Emission Service [15]. Based on participants' administrative residential codes, we identified and 
matched the nearest monitoring site to each residence at the district level. The 2-year average concentrations of each district-specific $\mathrm{PM}_{10}$ and $\mathrm{PM}_{2.5}$ exposure for the period of 2010 to 2011, corresponding to 2 years before the follow-up health examination visits between 2012 and 2013, were calculated using their daily mean values at a total of 49 national monitoring sites placed in the residential areas. Daily mean levels of coarse particulate matter $\left(\mathrm{PM}_{10-2.5}\right)$ were calculated by subtracting the daily mean levels of $\mathrm{PM}_{2.5}$ from those of $\mathrm{PM}_{10}$. For the analysis stratified by quantitative exposure to each $\mathrm{PM}_{10-2.5}$ and $\mathrm{PM}_{2.5}$, their 2-year mean concentrations were classified into four levels using quartiles.

\section{Main outcomes and other variables}

Information on participants' sociodemographic characteristics (age, sex, residential area, and income level) and the results of self-reported health questionnaires, physical examinations, and biochemical tests were gathered from the first and second biennial health examinations during the study period.

The main outcomes for this study were changes in the levels of fasting blood glucose (FBG) and lipid profiles 2 years after PM exposure, calculated by subtracting each laboratory value obtained during the first health examination (baseline) from those of the second health examination (follow-up). These blood samples were taken after fasting for at least $12 \mathrm{~h}$.

Data on systolic and diastolic blood pressure, anthropometric indices (height and weight), and health behaviours (smoking status, alcohol consumption, and physical activity) as well as sociodemographic information were collected from the first health examination records as covariates and baseline characteristics of the study population. Body mass index (BMI) was calculated by dividing weight in kilograms by the square of height in metres and categorized as underweight $\left(<18.5 \mathrm{~kg} / \mathrm{m}^{2}\right)$, normal (18.5 to $22.9 \mathrm{~kg} / \mathrm{m}^{2}$ ), overweight (23.0 to $24.9 \mathrm{~kg}$ / $\left.\mathrm{m}^{2}\right)$, or obese $\left(\geq 25.0 \mathrm{~kg} / \mathrm{m}^{2}\right)$ according to the Asia Pacific criteria of the World Health Organization [16]. Based on the responses of the self-reported questionnaire, we classified smoking status into 'never', 'ex-', and 'current' smokers. Drinking habits were categorized by the frequency of alcohol consumption per week. The physically 'active' group was defined as those who engaged in moderate activities for $\geq 30 \mathrm{~min}$ in a day, at least 5 days a week, or in vigorous activities for $\geq 20 \mathrm{~min}$ in a day, at least 3 days a week [17]. The physically 'inactive' group was defined as not meeting these criteria. The status of comorbid diseases for the past 10 years was assessed at baseline with the Charlson comorbidity index (CCI) developed by Quan et al., which has been used broadly in public health research [18].

\section{Statistical analysis}

All analyses were conducted for each estimate of $\mathrm{PM}_{10-}$ 2.5 and $\mathrm{PM}_{2.5}$ concentrations separately, and the participants were categorized into four groups by quartiles of the mean PM levels for the 2 years of the first health examination period. To compare between-group characteristics of the population, categorical variables were expressed as percentages of the population using Pearson chi-squared tests, while continuous variables were expressed as the means with their standard deviations using analysis of variance (ANOVA). Multiple linear regression analyses were conducted to estimate associations of exposure to PM with changes in FBG and lipid profiles at a 2-year interval after adjusting for potential confounding covariates, including age, sex, smoking status, alcohol consumption, physical activity, BMI, income level, and CCI. The associations were expressed as the adjusted mean differences of the change in laboratory outcome values with 95\% confidence interval (95\% CI) based on quartiles of PM exposure levels. We also performed stratified analyses based on the following characteristics of the participants at baseline: CCI score $(0$ versus $\geq 1)$; FBG levels $(<126 \mathrm{mg} / \mathrm{dL}$ versus $\geq 126 \mathrm{mg} / \mathrm{dL}$ ); LDL-C levels $(<160 \mathrm{mg} / \mathrm{dL}$ versus $\geq 160 \mathrm{mg} / \mathrm{dL})$; physical activity (active versus inactive) and age $(<60$ years versus $\geq 60$ years) as potential effect modifiers, by examining the interactions of the potential effect modifiers and $\mathrm{PM}_{2.5}$ exposure separately. To test the stability of the associations, we further conducted sensitivity analyses by excluding participants whose FBG, LDL-C, and blood pressure were equal to or higher than the normal range (FBG $\geq 126 \mathrm{mg} / \mathrm{dL}$, LDL-C $\geq 160 \mathrm{mg} / \mathrm{dL}$, and blood pressure $\geq 140 / 90 \mathrm{mmHg}$ ) in the heath examination at baseline. Additional sensitivity analyses were performed after excluding participants who had different residences from baseline over the study period. Statistical data mining and analyses were conducted using SAS version 9.4 (SAS Institute Inc., Cary, NC, USA) and STATA version 15.1 (Stata Corp., TX, USA), and $P$ values of $\leq 0.05$ were considered statistically significant.

\section{Results}

The mean concentrations of exposure to coarse PM $\left(\mathrm{PM}_{10-2.5}\right)$ and fine $\mathrm{PM}\left(\mathrm{PM}_{2.5}\right)$ at baseline were $22.81 \pm$ $4.64 \mu \mathrm{g} / \mathrm{m}^{3}$ and $25.94 \pm 3.56 \mu \mathrm{g} / \mathrm{m}^{3}$, respectively. Of the 85,869 participants, $50.77 \%$ were men, and $60.37 \%$ were aged 40-64 years. At baseline, each group, according to quartiles of $\mathrm{PM}_{10-2.5}$ and $\mathrm{PM}_{2.5}$, had similar proportions of participants regarding smoking, alcohol consumption, and physical activity status. The sociodemographic and clinical characteristics of the participants according to quartiles of each $\mathrm{PM}_{10-2.5}$ and $\mathrm{PM}_{2.5}$ at baseline are presented in Table 1. 
Table 1 Baseline characteristics of study population according to quartiles of each PM $10-2.5$ and PM 2.5 levels at individual's residential districts

\begin{tabular}{|c|c|c|c|c|c|c|c|c|c|c|c|c|}
\hline & \multirow{2}{*}{$\begin{array}{l}\text { Whole } \\
\text { NHIS-NSC }\end{array}$} & \multirow{2}{*}{$\begin{array}{l}\text { All study } \\
\text { population }\end{array}$} & \multicolumn{4}{|c|}{ Quartiles of $\mathrm{PM}_{10-2.5}$ levels } & \multirow[t]{2}{*}{$P$ value } & \multicolumn{4}{|c|}{ Quartiles of $\mathrm{PM}_{2.5}$ levels } & \multirow[t]{2}{*}{$P$ value } \\
\hline & & & Q1 & Q2 & Q3 & Q4 & & Q1 & Q2 & Q3 & Q4 & \\
\hline $\begin{array}{l}\text { Mean level of } \mathrm{PM}_{10-2.5} \\
\mu \mathrm{g} / \mathrm{m}^{3} \pm \mathrm{SD}\end{array}$ & & $\begin{array}{l}22.81 \\
\pm 4.64\end{array}$ & $\begin{array}{l}17.59 \\
\pm 3.67\end{array}$ & $\begin{array}{l}21.92 \\
\pm 0.66\end{array}$ & $\begin{array}{l}24.34 \\
\pm 0.83\end{array}$ & $\begin{array}{l}28.14 \\
\pm 3.17\end{array}$ & $<0.001$ & $\begin{array}{l}22.12 \\
\pm 5.49\end{array}$ & $\begin{array}{l}23.24 \\
\pm 2.68\end{array}$ & $\begin{array}{l}23.85 \\
\pm 3.87\end{array}$ & $\begin{array}{l}21.86 \\
\pm 5.73\end{array}$ & $<0.001$ \\
\hline $\begin{array}{l}\text { Mean level of } \mathrm{PM}_{2.5} \\
\mu \mathrm{g} / \mathrm{m}^{3} \pm \mathrm{SD}\end{array}$ & & $25.94 \pm 3.56$ & $\begin{array}{l}25.88 \\
\pm 4.18\end{array}$ & $\begin{array}{l}25.23 \\
\pm 1.97\end{array}$ & $\begin{array}{l}26.30 \\
\pm 2.83\end{array}$ & $\begin{array}{l}26.43 \\
\pm 4.47\end{array}$ & $<0.001$ & $\begin{array}{l}21.78 \\
\pm 1.83\end{array}$ & $\begin{array}{l}24.97 \\
\pm 0.62\end{array}$ & $\begin{array}{l}26.89 \\
\pm 0.70\end{array}$ & $\begin{array}{l}30.98 \\
\pm 2.15\end{array}$ & $<0.001$ \\
\hline Population, $n$ & $1,006,481$ & 85,869 & 23,354 & 22,292 & 19,114 & 21,109 & & 22,717 & 21,622 & 22,959 & 18,571 & \\
\hline Men, \% & 50.02 & 50.77 & 50.66 & 50.79 & 51.03 & 50.64 & 0.858 & 50.25 & 50.9 & 50.45 & 51.65 & 0.026 \\
\hline Age, \% & & & & & & & $<0.001$ & & & & & $<0.001$ \\
\hline $0-19$ years & 22.21 & - & & & & & & & & & & \\
\hline 20-39 years & 29.26 & 25.05 & 21.97 & 26.51 & 26.32 & 25.76 & & 22.76 & 28.01 & 26.2 & 22.97 & \\
\hline 40-64 years & 36.87 & 60.37 & 62.71 & 60.08 & 58.90 & 59.39 & & 62.2 & 57.24 & 59.14 & 63.28 & \\
\hline$\geq 65$ years & 11.66 & 14.59 & 15.32 & 13.40 & 14.78 & 14.85 & & 15.04 & 14.75 & 14.66 & 13.75 & \\
\hline Income level, \% & & & & & & & $<0.001$ & & & & & $<0.001$ \\
\hline Lowest & 31.85 & 27.40 & 28.42 & 26.61 & 27.33 & 27.19 & & 27.57 & 25.91 & 26.6 & 29.93 & \\
\hline Lower middle & 28.57 & 28.77 & 28.44 & 28.58 & 28.70 & 29.41 & & 28.34 & 27.37 & 29.01 & 30.64 & \\
\hline Higher middle & 25.18 & 28.25 & 28.77 & 28.18 & 27.37 & 28.53 & & 28.94 & 27.5 & 28.62 & 27.83 & \\
\hline Highest & 14.41 & 15.57 & 14.37 & 16.62 & 16.61 & 14.87 & & 15.15 & 19.23 & 15.78 & 11.6 & \\
\hline Smoking status, \% & & & & & & & 0.164 & & & & & $<0.001$ \\
\hline Never smoker & 62.10 & 62.82 & 62.37 & 63.00 & 62.67 & 63.28 & & 62.86 & 63.22 & 63.47 & 61.52 & \\
\hline Ex-smoker & 14.52 & 15.64 & 15.63 & 15.88 & 15.46 & 15.55 & & 15.83 & 16.14 & 15.57 & 14.89 & \\
\hline Current smoker & 23.38 & 21.54 & 22.00 & 21.13 & 21.87 & 21.17 & & 21.31 & 20.63 & 20.96 & 23.6 & \\
\hline $\begin{array}{l}\text { Alcohol } \\
\text { consumption, } \\
\text { per week, \% }\end{array}$ & & & & & & & $<0.001$ & & & & & $<0.001$ \\
\hline None & 53.03 & 51.93 & 53.39 & 50.89 & 50.90 & 52.35 & & 52.3 & 49.81 & 51.62 & 54.33 & \\
\hline $1-2$ times & 33.20 & 34.79 & 33.71 & 35.68 & 35.63 & 34.30 & & 34.37 & 36.28 & 35.01 & 33.33 & \\
\hline 3-4 times & 9.74 & 9.62 & 9.18 & 9.88 & 9.73 & 9.71 & & 9.67 & 10.17 & 9.84 & 8.63 & \\
\hline$\geq 5$ times & 4.02 & 3.66 & 3.72 & 3.55 & 3.74 & 3.64 & & 3.66 & 3.74 & 3.54 & 3.71 & \\
\hline Physical activity ${ }^{a}, \%$ & & & & & & & 0.055 & & & & & 0.053 \\
\hline Active & 30.00 & 20.53 & 21.00 & 20.34 & 19.98 & 20.70 & & 21.16 & 20.33 & 20.24 & 20.34 & \\
\hline Inactive & 70.00 & 79.47 & 79.00 & 79.66 & 80.02 & 79.30 & & 78.84 & 79.67 & 79.76 & 79.66 & \\
\hline $\begin{array}{l}\mathrm{BMI}^{b}, \mathrm{~kg} / \mathrm{m}^{2} \\
\text { mean } \pm \mathrm{SD}\end{array}$ & $23.74 \pm 3.26$ & $23.64 \pm 3.15$ & $\begin{array}{l}23.64 \\
\pm 3.12\end{array}$ & $\begin{array}{l}23.62 \\
\pm 3.17\end{array}$ & $\begin{array}{l}23.70 \\
\pm 3.16\end{array}$ & $\begin{array}{l}23.60 \\
\pm 3.15\end{array}$ & 0.011 & $23.69 \pm 3.15$ & $23.62 \pm 3.19$ & $23.65 \pm 3.16$ & $23.58 \pm 3.08$ & 0.002 \\
\hline Underweight, \% & 3.86 & 3.71 & 3.37 & 3.92 & 3.69 & 3.89 & 0.001 & 3.35 & 4.17 & 3.79 & 3.52 & $<0.001$ \\
\hline Normal, \% & 38.99 & 39.98 & 40.52 & 39.84 & 39.31 & 40.15 & & 39.81 & 39.7 & 39.77 & 40.78 & \\
\hline Overweight, \% & 24.60 & 25.33 & 25.38 & 25.53 & 24.91 & 25.43 & & 25.29 & 25.02 & 25.19 & 25.9 & \\
\hline Obese, \% & 32.55 & 30.98 & 30.74 & 30.71 & 32.09 & 30.53 & & 31.54 & 31.11 & 31.25 & 29.8 & \\
\hline \multicolumn{13}{|l|}{$\begin{array}{l}\mathrm{BP}, \mathrm{mmHg} \\
\text { mean } \pm \mathrm{SD}\end{array}$} \\
\hline Systolic BP & $\begin{array}{l}122.50 \\
\pm 15.15\end{array}$ & $\begin{array}{l}121.76 \\
\pm 14.90\end{array}$ & $\begin{array}{l}122.23 \\
\pm 14.93\end{array}$ & $\begin{array}{l}121.51 \\
\pm 14.95\end{array}$ & $\begin{array}{l}122.03 \\
\pm 15.11\end{array}$ & $\begin{array}{l}121.29 \\
\pm 14.61\end{array}$ & $<0.001$ & $\begin{array}{l}121.60 \\
\pm 14.66\end{array}$ & $\begin{array}{l}121.48 \\
\pm 15.03\end{array}$ & $\begin{array}{l}121.69 \\
\pm 15.00\end{array}$ & $\begin{array}{l}122.38 \\
\pm 14.91\end{array}$ & 0.001 \\
\hline Diastolic BP & $\begin{array}{l}76.19 \\
\pm 10.11\end{array}$ & $\begin{array}{l}75.56 \\
\pm 10.08\end{array}$ & $\begin{array}{l}75.71 \\
\pm 9.96\end{array}$ & $\begin{array}{l}75.40 \\
\pm 10.16\end{array}$ & $\begin{array}{l}75.64 \\
\pm 10.29\end{array}$ & $\begin{array}{l}75.51 \\
\pm 9.93\end{array}$ & 0.005 & $\begin{array}{l}75.52 \\
\pm 9.95\end{array}$ & $\begin{array}{l}75.45 \\
\pm 10.30\end{array}$ & $\begin{array}{l}75.57 \\
\pm 10.11\end{array}$ & $\begin{array}{l}75.73 \\
\pm 9.94\end{array}$ & 0.045 \\
\hline $\begin{array}{l}\mathrm{FBG}, \mathrm{mg} / \mathrm{dL}, \\
\text { mean } \pm \mathrm{SD}\end{array}$ & $\begin{array}{l}97.94 \\
\pm 24.08\end{array}$ & $\begin{array}{l}97.17 \\
\pm 22.82\end{array}$ & $\begin{array}{l}97.24 \\
\pm 23.34\end{array}$ & $\begin{array}{l}96.86 \\
\pm 21.93\end{array}$ & $\begin{array}{l}97.12 \\
\pm 22.92\end{array}$ & $\begin{array}{l}97.48 \\
\pm 23.08\end{array}$ & 0.039 & $\begin{array}{l}97.63 \\
\pm 22.55\end{array}$ & $\begin{array}{l}96.57 \\
\pm 22.20\end{array}$ & $\begin{array}{l}97.42 \\
\pm 22.63\end{array}$ & $\begin{array}{l}97.02 \\
\pm 24.06\end{array}$ & $<0.001$ \\
\hline $\begin{array}{l}\mathrm{TC}, \mathrm{mg} / \mathrm{dL}, \\
\text { mean } \pm \mathrm{SD}\end{array}$ & $\begin{array}{l}195.15 \\
\pm 37.05\end{array}$ & $\begin{array}{l}195.76 \\
\pm 36.41\end{array}$ & $\begin{array}{l}195.95 \\
\pm 36.70\end{array}$ & $\begin{array}{l}195.41 \\
\pm 36.05\end{array}$ & $\begin{array}{l}195.22 \\
\pm 36.18\end{array}$ & $\begin{array}{l}196.41 \\
\pm 36.64\end{array}$ & 0.003 & $\begin{array}{l}196.45 \\
\pm 36.62\end{array}$ & $\begin{array}{l}195.28 \\
\pm 35.99\end{array}$ & $\begin{array}{l}196.17 \\
\pm 36.25\end{array}$ & $\begin{array}{l}194.97 \\
\pm 36.79\end{array}$ & $<0.001$ \\
\hline $\begin{array}{l}\mathrm{TG}, \mathrm{mg} / \mathrm{dL} \\
\text { mean } \pm \mathrm{SD}\end{array}$ & $\begin{array}{l}132.83 \\
\pm 92.89\end{array}$ & $\begin{array}{l}128.58 \\
\pm 89.37\end{array}$ & $\begin{array}{r}129.89 \\
\pm 90.65\end{array}$ & $\begin{array}{l}127.75 \\
\pm 89.27\end{array}$ & $\begin{array}{l}130.62 \\
\pm 89.85\end{array}$ & $\begin{array}{l}126.15 \\
\pm 87.52\end{array}$ & $<0.001$ & $\begin{array}{l}128.03 \\
\pm 90.00\end{array}$ & $\begin{array}{l}127.77 \\
\pm 87.65\end{array}$ & $\begin{array}{l}127.58 \\
\pm 88.62\end{array}$ & $\begin{array}{l}131.43 \\
\pm 91.43\end{array}$ & $<0.001$ \\
\hline $\begin{array}{l}\mathrm{HDL}-\mathrm{C}, \mathrm{mg} / \mathrm{dL}, \\
\text { mean } \pm \mathrm{SD}\end{array}$ & $\begin{array}{l}55.84 \\
\pm 24.43\end{array}$ & $\begin{array}{l}55.76 \\
\pm 15.36\end{array}$ & $\begin{array}{l}55.73 \\
\pm 15.39\end{array}$ & $\begin{array}{l}55.83 \\
\pm 16.40\end{array}$ & $\begin{array}{l}55.83 \\
\pm 15.88\end{array}$ & $\begin{array}{l}55.68 \\
\pm 13.62\end{array}$ & 0.687 & $\begin{array}{l}55.80 \\
\pm 15.80\end{array}$ & $\begin{array}{l}55.69 \\
\pm 15.56\end{array}$ & $\begin{array}{l}55.69 \\
\pm 15.60\end{array}$ & $\begin{array}{l}55.90 \\
\pm 14.25\end{array}$ & 0.435 \\
\hline
\end{tabular}


Table 1 Baseline characteristics of study population according to quartiles of each $\mathrm{PM}_{10-2.5}$ and $\mathrm{PM}_{2.5}$ levels at individual's residential districts (Continued)

\begin{tabular}{|c|c|c|c|c|c|c|c|c|c|c|c|c|}
\hline & \multirow{2}{*}{$\begin{array}{l}\text { Whole } \\
\text { NHIS-NSC }\end{array}$} & \multirow{2}{*}{$\begin{array}{l}\text { All study } \\
\text { population }\end{array}$} & \multicolumn{4}{|c|}{ Quartiles of $\mathrm{PM}_{10-2.5}$ levels } & \multirow[t]{2}{*}{$P$ value } & \multicolumn{4}{|c|}{ Quartiles of $\mathrm{PM}_{2.5}$ levels } & \multirow[t]{2}{*}{$P$ value } \\
\hline & & & Q1 & Q2 & Q3 & Q4 & & Q1 & Q2 & Q3 & Q4 & \\
\hline $\begin{array}{l}\mathrm{LDL}-\mathrm{C}, \mathrm{mg} / \mathrm{dL} \\
\text { mean } \pm \mathrm{SD}\end{array}$ & $\begin{array}{l}114.06 \\
\pm 36.04\end{array}$ & $\begin{array}{l}114.71 \\
\pm 34.33\end{array}$ & $\begin{array}{l}114.76 \\
\pm 34.65\end{array}$ & $\begin{array}{l}114.45 \\
\pm 33.59\end{array}$ & $\begin{array}{l}113.82 \\
\pm 35.04\end{array}$ & $\begin{array}{l}115.74 \\
\pm 34.07\end{array}$ & $<0.001$ & $\begin{array}{l}115.57 \\
\pm 34.72\end{array}$ & $\begin{array}{l}114.28 \\
\pm 33.45\end{array}$ & $\begin{array}{l}115.55 \\
\pm 34.71\end{array}$ & $\begin{array}{l}113.13 \\
\pm 34.32\end{array}$ & $<0.001$ \\
\hline $\mathrm{CCl}, \%$ & & & & & & & $<0.001$ & & & & & $<0.001$ \\
\hline 0 & 33.87 & 28.83 & 27.09 & 29.08 & 30.29 & 29.17 & & 27.71 & 30.96 & 28.64 & 27.97 & \\
\hline 1 & 38.19 & 31.92 & 31.66 & 31.89 & 31.76 & 32.39 & & 31.23 & 31.69 & 32.02 & 32.92 & \\
\hline$\geq 2$ & 27.94 & 39.25 & 41.25 & 39.03 & 37.95 & 38.43 & & 41.07 & 37.35 & 39.34 & 39.11 & \\
\hline
\end{tabular}

Note: $\mathrm{PM}_{10-2.5}$, particulate matter sized 2.5-10 $\mu \mathrm{m}$ in aerodynamic diameter; $\mathrm{PM}_{2.5}$, particulate matter with aerodynamic diameter $\leq 2.5 \mu$ m; $N H I S-N S C$ National Health Insurance Service-National Sample Cohort, $n$, number, SD standard deviation, BMI body mass index, BP blood pressure, $F B G$ fasting blood glucose, TC total cholesterol, TG triglycerides, $H D L-C$ high-density lipoprotein cholesterol, $L D L-C$ low-density lipoprotein cholesterol, $C C I C$ harlson comorbidity index

${ }^{a}$ Participants were divided into active or inactive group based on the total minutes of each level of physical activities. Active group was specified as performing for $\geq 30 \mathrm{~min}$ in a day at least 5 days a week in moderate activities, or for $\geq 20 \mathrm{~min}$ in a day at least 3 days a week in vigorous activities

${ }^{b}$ Participants were categorized as underweight, normal, overweight, and obese by Asia pacific criteria of World Health Organization

We found no significant correlations of the changes in FBG and all the lipid profiles, including total cholesterol (TC), triglycerides (TG), high-density lipoprotein cholesterol (HDL-C), and low-density lipoprotein cholesterol (LDL-C), with increases in $\mathrm{PM}_{10-2.5}$ concentrations at 2year intervals after adjusting for other covariates $(P$ for trend $\geq 0.05$ for each of FBG, TC, TG, HDL-C, and LDL$\mathrm{C})$. When assessing the associations of $\mathrm{PM}_{2.5}$ exposure and the changes in laboratory values, we observed a positive correlation of elevations of both FBG and LDL$C$ levels ( $P$ for trend $=0.015$ and 0.010 , respectively) after adjusting for other covariates. However, there was no significant association of an increase in exposure to $\mathrm{PM}_{2.5}$ with changes in TC, TG, and HDL-C levels ( $P$ for trend $\geq 0.05$ for TC, TG, and HDL-C). The associations of $\mathrm{PM}_{10-2.5}$ and $\mathrm{PM}_{2.5}$ exposure with changes in FBG and lipid profiles are shown in Table 2.

The results of stratified analyses are presented in Table 3 . Significant modified effects were observed for the physical activity status and age. We found that significant elevations in FBG and LDL-C levels were associated with increments of $\mathrm{PM}_{2.5}$ exposure in all subgroups, except the groups with $\mathrm{FBG} \geq 126 \mathrm{mg} / \mathrm{dL}$, LDL-C $\geq 160 \mathrm{mg} / \mathrm{dL}$, or physically active groups at baseline. In association with $\mathrm{PM}_{2.5}$ exposure for age-specific groups, significant changes in FBG and LDL-C levels were found only among groups aged $\geq 60$ years $(P$ for trend $=0.014$ and 0.004 , respectively). In the sensitivity analyses, no substantial change was observed from the main analysis, and the associations of $\mathrm{PM}_{2.5}$ exposure with increases in $\mathrm{FBG}$ and LDL-C levels remained positive and significant among participants whose FBG, LDL-C, and blood pressure at baseline were within the normal range $(P$ for trend $<0.001$ for FBG and 0.028 for LDL-C, respectively). Restricting the analyses to participants who had the same addresses over the study period also showed the significant associations of $\mathrm{PM}_{2.5}$ exposure with increases in FBG and LDL-C levels $(P$ for trend $=0.006$ for FBG and 0.019 for LDL-C, respectively).

\section{Discussion}

In this study, we evaluated the associations of exposure to ambient $\mathrm{PM}_{10-2.5}$ and $\mathrm{PM}_{2.5}$ with changes in the levels of fasting glucose and lipid profiles at 2-year intervals in a representative Korean population using a nationwide cohort. There was a significant relationship of higher concentrations of ambient $\mathrm{PM}_{2.5}$ based on an individual's residential district with elevations in FBG and LDL-C levels after 2 years of exposure among the study population. However, no association of $\mathrm{PM}_{10-2.5}$ levels, as coarse particles, with changes in FBG and lipid profiles was found in this study. To our knowledge, this is the first study to provide direct evidence of the sizespecific effects of PM in Asia by evaluating the adverse metabolic effects of both fine and coarse PM on changes in laboratory values.

Several studies provide strong evidence that shows that increases in ambient PM levels lead to increased incidence rates of metabolic diseases, which include but are not limited to diabetes and dyslipidaemia [19-21]. Furthermore, another study by Yitshak Sade et al. suggested that increases in the levels of FBG, glycosylated haemoglobin, and LDL-C are associated with increase in $\mathrm{PM}_{10}$ and $\mathrm{PM}_{2.5}$ concentrations at 3-month intervals from exposure [22]. Similarly, this study provides strong evidence for elevated FBG and LDL-C levels when exposed to $\mathrm{PM}_{2.5}$ at 2-year intervals with large-scale demographics. The well-established mechanism of PM- associated metabolic changes in glucose or lipids has not been clarified. Possible explanations for the observed metabolic adverse effects of PM might include systemic inflammation related to activated pro-inflammatory cytokines and subsequent oxidative stress, as well as endothelial dysfunction. A previous epidemiological study of children showed a higher BMI among those who lived in areas with high levels of air pollution, including PM exposure [23]. It was suggested that exposure to PM may affect the deposition of visceral adipose tissue $[24,25]$. The impaired lipid metabolism seems to 
Table 2 Association between each $\mathrm{PM}_{10-2.5}$ and $\mathrm{PM}_{2.5}$ levels and changes in individual's concentration of fasting glucose and lipid profiles

\begin{tabular}{|c|c|c|c|c|c|}
\hline & \multicolumn{4}{|c|}{ Quartiles of $\mathrm{PM}_{10-2.5}$ levels } & \multirow[t]{2}{*}{$P$ for trends } \\
\hline & Q1 & Q2 & Q3 & Q4 & \\
\hline Mean level, $\mu \mathrm{g} / \mathrm{m}^{3} \pm \mathrm{SD}$ & $17.59 \pm 3.67$ & $21.92 \pm 0.66$ & $24.34 \pm 0.83$ & $28.14 \pm 3.17$ & \\
\hline \multicolumn{6}{|c|}{ Mean difference of individual's concentration, mg/dL (95\% Cl) } \\
\hline \multirow[t]{2}{*}{ FBG } & 0.956 & 0.588 & 1.214 & 0.598 & 0.429 \\
\hline & $(0.693-1.218)$ & $(0.318-0.857)$ & $(0.923-1.505)$ & $(0.322-0.875)$ & \\
\hline \multirow[t]{2}{*}{ TC } & 0.446 & 0.175 & 0.316 & 0.408 & 0.998 \\
\hline & $(0.027-0.864)$ & $(-0.254-0.605)$ & $(-0.147-0.779)$ & $(-0.032-0.848)$ & \\
\hline \multirow[t]{2}{*}{ TG } & -0.504 & -0.322 & -0.743 & 0.023 & 0.620 \\
\hline & $(-1.518-0.510)$ & $(-1.363-0.718)$ & $(-1.865-0.380)$ & $(-1.044-1.090)$ & \\
\hline \multirow[t]{2}{*}{$\mathrm{HDL}-\mathrm{C}$} & -0.034 & -0.116 & -0.350 & -0.146 & 0.214 \\
\hline & $(-0.228-0.161)$ & $(-0.315-0.083)$ & $(-0.565--0.135)$ & $(-0.350-0.059)$ & \\
\hline \multirow[t]{4}{*}{ LDL-C } & 0.669 & 0.361 & 0.587 & 0.907 & 0.360 \\
\hline & $(0.248-1.090)$ & $(-0.071-0.793)$ & $(0.122-1.052)$ & $(0.464-1.351)$ & \\
\hline & \multicolumn{4}{|c|}{ Quartiles of $\mathrm{PM}_{2.5}$ levels } & $P$ for trends \\
\hline & Q1 & Q2 & Q3 & Q4 & \\
\hline Mean level, $\mu \mathrm{g} / \mathrm{m}^{3} \pm \mathrm{SD}$ & $21.78 \pm 1.83$ & $24.97 \pm 0.62$ & $26.89 \pm 0.70$ & $30.98 \pm 2.15$ & \\
\hline \multicolumn{6}{|c|}{ Mean difference of individual's concentration, mg/dL ( $95 \% \mathrm{Cl}$ ) } \\
\hline \multirow[t]{2}{*}{ FBG } & 0.550 & 0.975 & 0.687 & $1.180^{*}$ & 0.015 \\
\hline & $(0.283-0.816)$ & $(0.702-1.249)$ & $(0.422-0.953)$ & $(0.886-1.475)$ & \\
\hline \multirow[t]{2}{*}{ TC } & 0.192 & 0.46 & 0.076 & 0.696 & 0.312 \\
\hline & $(-0.232-0.616)$ & $(0.024-0.896)$ & $(-0.347-0.498)$ & $(0.227-1.166)$ & \\
\hline \multirow[t]{2}{*}{ TG } & -0.404 & -0.524 & -0.235 & -0.365 & 0.862 \\
\hline & $(-1.432-0.625)$ & $(-1.581-0.533)$ & $(-1.259-0.790)$ & $(-1.505-0.774)$ & \\
\hline \multirow[t]{2}{*}{ HDL-C } & -0.219 & 0.026 & -0.208 & -0.212 & 0.690 \\
\hline & $(-0.416--0.022)$ & $(-0.176-0.229)$ & $(-0.404--0.012)$ & $(-0.430-0.006)$ & \\
\hline \multirow[t]{2}{*}{ LDL-C } & 0.341 & 0.618 & 0.316 & $1.384^{*}$ & 0.010 \\
\hline & $(-0.086-0.768)$ & $(0.180-1.056)$ & $(-0.109-0.741)$ & $(0.910-1.857)$ & \\
\hline
\end{tabular}

Note: Models were adjusted for age, sex, income level, smoking status, alcohol consumption status, $B M I$ physical activity, and $C C I$ score. $P M_{10-2.5}$, particulate matter sized 2.5-10 $\mu \mathrm{m}$ in aerodynamic diameter, $P M_{2.5}$ particulate matter with aerodynamic diameter $\leq 2.5 \mu \mathrm{m} ; \mathrm{n}$, number, $S D$ standard deviation, $C l$ confidential interval, FBG fasting blood glucose, TC total cholesterol, TG triglycerides, HDL-C high-density lipoprotein cholesterol, $L D L-C$ low-density lipoprotein cholesterol

* $P$ value $<0.05$ of multiple regression for quartile 4 group, compared to quartile 1 group as a reference

be related to oxidative stress and systemic inflammation through pro-inflammatory cytokine release induced by the accumulation and impairment of visceral adipose tissue by PM exposure $[10,26]$. Sun et al. suggested endothelial dysfunction as another explanatory mechanism of these PM-associated cardiometabolic changes, which was found in animal models [27]. It may also help explain the development of cardiovascular morbidity, as well as cardiometabolic risks in association with PM exposure [28]. For changes in TC and TG levels, no significant relationship with PM exposure was obtained after adjusting for covariates in this study. Previous studies have suggested that higher concentrations of ambient PM were correlated with lower concentrations and impaired functions of HDL-C, as well as poor health outcomes of TC and TG concentrations [22, 29-31]. However, their long-term effects could not be identified clearly and were inconsistent regarding the relationship between PM and lipid profiles, as most were conducted during short-term periods. More studies are needed to identify the exposure periods that show metabolic effects and their mechanism in the future.

We found no significant relationship between $\mathrm{PM}_{10}$ 2.5, which comprises more coarse components than $\mathrm{PM}_{2.5}$, and changes in FBG and lipid profile levels. This finding suggests that PM may contribute to the development of diabetes and dyslipidaemia at a disproportional intensity between $\mathrm{PM}_{10-2.5}$ and $\mathrm{PM}_{2.5}$. It was well documented that long-term exposure to $\mathrm{PM}_{2.5}$ exerted more remarkable and negative health effects on morbidity and 
Table 3 Stratified analyses of association between $\mathrm{PM}_{2.5}$ concentrations and change in levels of fasting glucose and lipid profiles

\begin{tabular}{|c|c|c|c|c|c|c|c|}
\hline & \multirow{2}{*}{$\begin{array}{l}\text { Mean difference } \\
\text { of individual's } \\
\text { concentration, } \\
\text { mg/dL ( } 95 \% \text { Cl) }\end{array}$} & \multicolumn{4}{|c|}{ Quartiles of $\mathrm{PM}_{2.5}$ levels } & \multirow{2}{*}{$\begin{array}{l}P \text { for } \\
\text { trends }\end{array}$} & \multirow[t]{2}{*}{$P_{\text {inter }}$} \\
\hline & & Q1 & Q2 & Q3 & Q4 & & \\
\hline $\mathrm{CCl}$ & & & & & & & 0.207 \\
\hline \multirow[t]{3}{*}{0} & Mean level, $\mu \mathrm{g} / \mathrm{m}^{3} \pm \mathrm{SD}$ & $21.839 \pm 1.790$ & $24.888 \pm 0.574$ & $26.609 \pm 0.513$ & $30.563 \pm 2.221$ & & \\
\hline & FBG & $\begin{array}{l}0.892 \\
(0.491-1.293)\end{array}$ & $\begin{array}{l}0.940 \\
(0.536-1.345)\end{array}$ & $\begin{array}{l}1.082 \\
(0.675-1.490)\end{array}$ & $\begin{array}{l}1.928 \\
(1.520-2.337)\end{array}$ & $<0.001$ & \\
\hline & LDL-C & $\begin{array}{l}1.437 \\
(0.703-2.171)\end{array}$ & $\begin{array}{l}1.924 \\
(1.185-2.664)\end{array}$ & $\begin{array}{l}2.077 \\
(1.332-2.823)\end{array}$ & $\begin{array}{l}2.934 \\
(2.183-3.684)\end{array}$ & 0.006 & \\
\hline \multirow[t]{3}{*}{$\geq 1$} & Mean level, $\mu \mathrm{g} / \mathrm{m}^{3} \pm \mathrm{SD}$ & $21.779 \pm 1.834$ & $24.972 \pm 0.621$ & $26.890 \pm 0.701$ & $30.979 \pm 2.148$ & & \\
\hline & FBG & $\begin{array}{l}0.550 \\
(0.283-0.816)\end{array}$ & $\begin{array}{l}0.975 \\
(0.702-1.249)\end{array}$ & $\begin{array}{l}0.687 \\
(0.422-0.953)\end{array}$ & $\begin{array}{l}1.180 \\
(0.886-1.475)\end{array}$ & 0.015 & \\
\hline & LDL-C & $\begin{array}{l}0.341 \\
(-0.086-0.768)\end{array}$ & $\begin{array}{l}0.618 \\
(0.180-1.056)\end{array}$ & $\begin{array}{l}0.316 \\
(-0.109-0.741)\end{array}$ & $\begin{array}{l}1.384 \\
(0.910-1.857)\end{array}$ & 0.010 & \\
\hline
\end{tabular}

FBG at baseline, mg/dL

$<126$

$\geq 126$

$$
\text { Mean level, } \mu \mathrm{g} / \mathrm{m}^{3} \pm \mathrm{SD}
$$
FBG

LDL-C

$21.779 \pm 1.832$

1.711

(1.506-1.915)

0.825

(0.395-1.255)

Mean level, $\mu \mathrm{g} / \mathrm{m}^{3} \pm \mathrm{SD}$

FBG

LDL-C

$21.776 \pm 1.866$

$-18.051$

(-20.907--15.196)

$-7.618$

(-9.899--5.337)

LDL-C at baseline, $\mathrm{mg} / \mathrm{dL}$

$$
<160
$$

$\geq 160$

Mean level, $\mu \mathrm{g} / \mathrm{m}^{3} \pm \mathrm{SD}$

FBG

$21.786 \pm 1.832$

0.592

(0.314-0.871)

LDL-C

$$
3.519
$$

$$
\text { (3.127-3.910) }
$$

Mean level, $\mu \mathrm{g} / \mathrm{m}^{3} \pm \mathrm{SD}$

$21.731 \pm 1.845$

FBG

0.295

(-0.602-1.192)

LDL-C

Physical activity ${ }^{a}$

Active

Inactive

Mean level, $\mu \mathrm{g} / \mathrm{m}^{3} \pm \mathrm{SD}$

FBG

$-30.200$

$(-32.319--28.082)$

$24.972 \pm 0.619$

2.120

(1.910-2.329)

0.947

(0.507-1.388)

$24.972 \pm 0.650$

$-17.233$

$(-20.279--14.187)$

$-4.763$

$(-7.186--2.340)$

$(-7$

(-34.729--29.829)

$30.970 \pm 2.139$

2.636

(2.409-2.862)

1.767

(1.289-2.244)

$31.107 \pm 2.290$

$-21.123$

(-24.260--17.986)

$-4.444$

(-6.956--1.931)

0.152

$<0.001$

0.022

0.176

0.105

$30.964 \pm 2.137$

1.237

(0.930-1.544)

4.605

(4.172-5.037)

$31.157 \pm 2.279$

0.342

(-0.694-1.378)

0.986

0.366

0.032

$\begin{array}{ll}24.883 \pm 0.578 & 26.842 \pm 0.733 \\ 1.354 & 0.613 \\ (0.691-2.017) & (0.019-1.207) \\ 0.748 & 0.380 \\ (-0.246-1.742) & (-0.513-1.274) \\ 24.973 \pm 0.621 & 26.884 \pm 0.621 \\ 0.854 & 0.738 \\ (0.550-1.157) & (0.444-1.032) \\ 0.577 & 0.328 \\ (0.083-1.070) & (-0.151-0.807)\end{array}$

$31.094+2263$

1.092

(0.409-1.775)

0.629

1.425

(0.397-2.454)

0.271

$30.949 \pm 2.117$

1.210

(0.883-1.536)

1.353

(0.819-1.887)

0.009

0.021

$(-0.161-0.805)$

(0.083-1.070)

$24.969+0.612$

$26.859 \pm 0.686$

$30.953 \pm 2.133$

1.130

(0.786-1.475) 
Table 3 Stratified analyses of association between $\mathrm{PM}_{2.5}$ concentrations and change in levels of fasting glucose and lipid profiles (Continued)

\begin{tabular}{|c|c|c|c|c|c|c|}
\hline & \multirow{2}{*}{$\begin{array}{l}\text { Mean difference } \\
\text { of individual's } \\
\text { concentration, } \\
\text { mg/dL }(95 \% \text { Cl) }\end{array}$} & \multicolumn{4}{|c|}{ Quartiles of $\mathrm{PM}_{2.5}$ levels } & \multirow{2}{*}{$\begin{array}{l}P \text { for } \\
\text { trends }\end{array}$} \\
\hline & & Q1 & Q2 & Q3 & Q4 & \\
\hline & LDL-C & $\begin{array}{l}1.932 \\
(1.413-2.452)\end{array}$ & $\begin{array}{l}.085 \\
(1.560-2.610)\end{array}$ & $\begin{array}{l}1.606 \\
(1.092-2.120)\end{array}$ & $\begin{array}{l}2.512 \\
(1.945-3.078)\end{array}$ & 0.377 \\
\hline \multirow[t]{3}{*}{$\geq 60$} & Mean level, $\mu \mathrm{g} / \mathrm{m}^{3} \pm \mathrm{SD}$ & $21.754 \pm 1.858$ & $24.977 \pm 0.637$ & $26.941 \pm 0.723$ & $31.024 \pm 2.176$ & \\
\hline & FBG & $\begin{array}{l}0.049 \\
(-0.426-0.524)\end{array}$ & $\begin{array}{l}0.707 \\
(0.207-1.208)\end{array}$ & $\begin{array}{l}0.096 \\
(-0.383-0.574)\end{array}$ & $\begin{array}{l}1.259 \\
(0.717-1.802)\end{array}$ & 0.014 \\
\hline & LDL-C & $\begin{array}{l}-2.344 \\
(-3.081--1.607)\end{array}$ & $\begin{array}{l}-1.810 \\
(-2.587--1.033)\end{array}$ & $\begin{array}{l}-1.824 \\
(-2.568--1.080)\end{array}$ & $\begin{array}{l}-0.530 \\
(-1.374-0.315)\end{array}$ & 0.004 \\
\hline
\end{tabular}

Note: Models were adjusted for age, sex, income level, smoking status, alcohol consumption status, $B M I$ physical activity, and $C C /$ score. $P M_{2.5}$, particulate matter with aerodynamic diameter $\leq 2.5 \mu \mathrm{m} ; n$, number, $S D$ standard deviation, $B M I$ body mass index, $B P$ blood pressure, $F B G$ fasting blood glucose, $L D L-C$ low-density lipoprotein cholesterol, $\mathrm{CCl}$ Charlson comorbidity index, $P_{\text {inter, }} P$ value for the interaction terms

${ }^{a}$ Participants were divided into active or inactive group based on the total minutes of each level of physical activities. Active group was specified as performing for $\geq 30 \mathrm{~min}$ in a day at least 5 days a week in moderate activities, or for $\geq 20 \mathrm{~min}$ in a day at least 3 days a week in vigorous activities

mortality to some organ systems in humans compared with $\mathrm{PM}_{10}$ or $\mathrm{PM}_{10-2.5}$ exposure [32-36]. However, few studies on the size-specific metabolic effects of PM have been conducted, particularly in Asia. $\mathrm{PM}_{2.5}$ consists of smaller particles that can more easily penetrate and move into the lungs and vessels and has more pollutants emitted from anthropogenic sources such as traffic, industrial processes, or combustion of fuels than those of $\mathrm{PM}_{10-2.5}$ [33]. It induces more systemic and harmful health effects on the body with a larger surface and more toxicity compared with $\mathrm{PM}_{10-2.5}$. Similarly, our study obtained different results by diameter of PM, indicating a significant association of individual elevation in FBG and lipid levels with higher levels of $\mathrm{PM}_{2.5}$ in 2 years after exposure. In previous studies, it was suggested that $\mathrm{PM}_{2.5}$ exposure levels were correlated with changes in insulin sensitivity and systemic inflammation [37, 38]. $\mathrm{PM}_{2.5}$ may have more evident adverse effects on systemic metabolic regulation than $\mathrm{PM}_{10-2.5}$, by inducing impairment of pancreatic $\beta$-cell function or aggravation of insulin resistance and metabolic dysregulation, particularly at 2-year intervals from exposure.

We observed significant modifying effects of physical activity and age on the associations of ambient $\mathrm{PM}_{2.5}$ exposure with changes in FBG and LDL-C levels. It was showed weaker associations of PM with elevations in FBG and LDL-C levels among the physically active group. It is well known that physical inactivity increases the risk of many non-communicable diseases including cardiovascular diseases and type 2 diabetes with strong evidence, potentially leading to stiffening of the arteries $[39,40]$. Regular physical exercise reduces overall cardiometabolic risk, although some physiological reactions to exercise over a few hours or days may transiently cause a greater degree of PM deposition within the lungs by rapid and deep inhalation [41, 42]. Therefore, our findings highlight the potential protective effects of regular physical activity against the development of diabetes and dyslipidaemia in association with exposure to fine PM. Regular exercise appears to help attenuate the harmful cardiometabolic effects in association with ambient PM air pollution, according to the data. We also found that the associations of ambient $\mathrm{PM}_{2.5}$ exposure with changes in FBG and LDL-C levels were more pronounced among individuals aged $\geq 60$ years in this study. This finding validates that the elderly population may be more vulnerable to the harmful effects of air pollution, particularly $\mathrm{PM}_{2.5}$ exposure, given the evidence that susceptible groups with chronic diseases or the elderly had a higher risk of air pollution-related detrimental health effects than others [36, 43, 44].

Our study indicated discrepancies in the significance of associations between fine PM and alterations in FBG and lipid profiles according to their baseline laboratory values among sub-groups. It was found that the group with a normal range of FBG or LDL-C had a stronger correlation with concentrations of $\mathrm{PM}_{2.5}$ exposure than the groups with higher concentrations of FBG or LDLC. This may be explained by the latent poor metabolic conditions with uncollected medication histories as potential confounding factors in the groups with abnormal laboratory values. The results from sub-groups according to CCI scores were consistent and support the sensitivity of the findings in this study.

Although the study remains reliable, a few limitations should be considered. Assessments of exposure to air pollutants were conducted using data from residential area-specific monitoring sites, which were the nearest stations from individual addresses available at the district level, although the area specific monitoring sites are reliable because the characteristics of the Korean Peninsula near areas may have varying PM data. In addition, we identified and matched the nearest monitoring site to each residence based on baseline records. Thus, the 
analyses were conducted under the assumption that the proportion of participants who had different addresses from baseline over the study period was negligible. It is recommended that in future studies, long-term exposure should be measured with fine-scale address data, considering changes in participants' residences over the study period. We calculated and used the levels of $\mathrm{PM}_{10-2.5}$ by subtracting $\mathrm{PM}_{2.5}$ from $\mathrm{PM}_{10}$ to compare the effects of fine PM with those of coarse particles due to the unavailability of measured data on $\mathrm{PM}_{10-2.5}$ using monitoring systems [45]. This estimation may induce a larger random error in measurements, but it would be unlikely to affect the statistical power for evaluating the $\mathrm{PM}_{10-25}$ effects with exposure estimates of individuals on a large scale of samples. Although we adjusted for several potential confounders, the possibility of other confounders from unmeasured variables cannot be completely ruled out, and the observed associations were likely to have been affected in part by residual confounding factors. Furthermore, the information on medication use was uncollectable due to privacy reasons, and it might have resulted in under- or over-estimation. These confounders need to be addressed to verify these results in further studies. Due to the characteristics of selfreported values in a few of our data, there may be possible response bias. We cannot conclude a direct relationship between ambient PM exposure and laboratory changes, as our study included an observational retrospective design. Thus, we cannot determine whether PM exposure actually affects changes in laboratory values. However, this concern might be mitigated by our large sample size, increasing the statistical power. Health effects of PM exposure may occur across various time periods over the lifetime. Thus, there may be a critical exposure period that results in particularly large health effects of PM, which is an important consideration for future studies.

Despite these limitations, to the best of our knowledge, this is the first study to evaluate the associations of each level of exposure to ambient $\mathrm{PM}_{10-2.5}$ and $\mathrm{PM}_{2.5}$ with mean changes in an individual's laboratory cardiometabolic risks, including fasting glucose and lipid profiles, at 2-year intervals using nationwide cohort data obtained from a large representative Korean population. Few studies have evaluated the effects of size-specific PM on changes in laboratory values, as well as their mechanisms and management. Thus, more studies are needed to identify or prevent this avoidable risk factor.

\section{Conclusions}

In conclusion, this study verifies that higher levels of ambient fine PM exposure were associated with elevated FBG and LDL-C levels in Korean adults. Our findings can help to explain the increased risk of cardiovascular disease and diabetes in areas with higher levels of air pollution and the stronger adverse effects of fine PM on human health than coarse PM. This study highlights the importance of consideration of the size-specific PMrelated health effects and public policies targeted at vulnerable groups, including older or physically inactive individuals, to reduce PM-associated harmful effects.

\section{Abbreviations \\ PM: Particulate matter; NHIS-NSC: National Health Insurance Service-National Sample Cohort; $\mathrm{PM}_{10}$ : Particulate matter with aerodynamic diameter $\leq 10 \mu \mathrm{m}$; $\mathrm{PM}_{2.5}$ : Particulate matter with aerodynamic diameter $\leq 2.5 \mu \mathrm{m} ; \mathrm{PM}_{10-}$ 25: Coarse particulate matter; FBG: Fasting blood glucose; BMl: Body mass index; CCl: Charlson comorbidity index; 95\% Cl: 95\% confidence interval; TC: Total cholesterol; TG: Triglycerides; HDL-C: High-density lipoprotein cholesterol; LDL-C: Low-density lipoprotein cholesterol}

\section{Acknowledgements}

Not applicable.

\section{Authors' contributions}

WS, JK, GL, and SP designed the study and directed its implementation. SC and SK collected the data. WS, GL, SC, YH, and SP analyzed and interpreted the data. WS, JK, GL, and SP wrote the manuscript. All authors read and approved the final manuscript.

\section{Funding}

Not applicable.

\section{Availability of data and materials}

The data that support the findings of this study are available from the NHISNSC but restrictions apply to the availability of these data due to ethical concerns, which were used under license for the current study, and so are not publicly available.

\section{Ethics approval and consent to participate}

The study protocol was approved by the ethics committee of Seoul National University (IRB number: E-1803-045-928). Individual written informed consent was obtained for the NHIS-NSC from all participants.

\section{Consent for publication}

Not applicable.

\section{Competing interests}

The authors declare that they have no competing interests.

\section{Author details}

${ }^{1}$ Department of Family Medicine, Chung-ang University Medical Center, Seoul 06973, Republic of Korea. ${ }^{2}$ Department of Family Medicine and Biomedical Sciences, College of Medicine, Seoul National University, 101 Daehak-ro, Jongno-gu, Seoul 03080, Republic of Korea. ${ }^{3}$ Department of Biomedical Sciences, College of Medicine, Seoul National University, Seoul 03087, Republic of Korea. ${ }^{4}$ Department of Medicine, College of Medicine, Seoul National University, Seoul 03087, Republic of Korea. ${ }^{5}$ Department of Preventive Medicine, College of Medicine, Seoul National University, Seoul 03087, Republic of Korea. ${ }^{6}$ Institute of Environmental Medicine, Seoul National University Medical Research Center, Seoul 03087, Republic of Korea. ${ }^{7}$ Environmental Health Center, College of Medicine, Seoul National University, Seoul 03087, Republic of Korea.

Received: 1 October 2019 Accepted: 11 March 2020

Published online: 03 April 2020

\section{References}

1. Naghavi M, Abajobir AA, Abbafati C, Abbas KM, Abd-Allah F, Abera SF, Aboyans V, Adetokunboh O, Afshin A, Agrawal A, et al. Global, regional, and national age-sex specific mortality for 264 causes of death, 1980-2016: a systematic analysis for the global burden of disease study 2016. Lancet. 2017;390(10100):1151-210. 
2. Ministry of Health and Welfare. Korea Health Statistics 2016: Korea National Health and nutrition examination survey. Cheongwon: Korea Centers of Disease Control and Prevention. p. 2017

3. Committee for the Korean Guidelines for the Management of Dyslipidemia. 2015 Korean guidelines for the Management of Dyslipidemia: executive summary (English translation). Korean Circ J. 2016:46(3):275-306.

4. Kim SE, Honda Y, Hashizume M, Kan H, Lim YH, Lee H, Kim CT, Yi SM, Kim H. Seasonal analysis of the short-term effects of air pollution on daily mortality in Northeast Asia. Sci Total Environ. 2017;576:850-7.

5. Dockery DW, Pope CA 3rd, Xu X, Spengler JD, Ware JH, Fay ME, Ferris BG Jr, Speizer FE. An association between air pollution and mortality in six U.S. cities. N Engl J Med. 1993;329(24):1753-9.

6. WHO air quality guidelines for particulate matter, ozone, nitrogen dioxide and sulfur dioxide [http://whqlibdoc.who.int/hq/2006/WHO_SDE_PHE_ OEH_06.02_eng.pdf].

7. Achilleos S, Kioumourtzoglou MA, Wu CD, Schwartz JD, Koutrakis P, Papatheodorou SI. Acute effects of fine particulate matter constituents on mortality: a systematic review and meta-regression analysis. Environ Int. 2017;109:89-100.

8. Park HY, Bae S, Hong Y-C. PM10 exposure and non-accidental mortality in Asian populations: a meta-analysis of time-series and case-crossover studies. J Prev Med Public Health. 2013;46(1):10-8.

9. Shin J, Choi J, Kim KJ. Association between long-term exposure of ambient air pollutants and cardiometabolic diseases: a 2012 Korean community health survey. Nutr Metab Cardiovasc Dis. 2019;29(2):144-51.

10. Thurston GD, Kipen H, Annesi-Maesano I, Balmes J, Brook RD, Cromar K, De Matteis S, Forastiere F, Forsberg B, Frampton MW, et al. A joint ERS/ATS policy statement: what constitutes an adverse health effect of air pollution? An analytical framework. Eur Respir J. 2017;49(1):1600419.

11. Forouzanfar $M$, Alexander $L$, Anderson $H$, Bachman V, Biryukov $S$, Brauer $M$, Burnett R, Casey D, Coates M, Cohen A. GBD 2013 risk factors collaborators. Global, regional, and national comparative risk assessment of 79 behavioural, environmental and occupational, and metabolic risks or clusters of risks in 188 countries, 1990-2013: a systematic analysis for the global burden of disease study 2013. Lancet. 2015;386(10010):2287-323.

12. Lee J, Lee JS, Park SH, Shin SA, Kim K. Cohort profile: the National Health Insurance Service-National Sample Cohort (NHIS-NSC), South Korea. Int J Epidemiol. 2017;46(2):e15.

13. Cohen Hubal EA, Sheldon LS, Burke JM, McCurdy TR, Berry MR, Rigas ML, Zartarian VG, Freeman N. Children's exposure assessment: a review of factors influencing Children's exposure, and the data available to characterize and assess that exposure. Environ Health Perspect. 2000;108(6): 475-86.

14. Kim ST. Quality control of air pollution monitoring system and establishment of data evaluation scheme(1). Incheon: Korean Air Pollutants Emission Service; 2015

15. National Institute of Environmental Research. Standard methods for the examination of air pollution. Incheon: National Institute of Environmental Research; 2017.

16. S C. The Asia-Pacific perspective: redefining obesity and its treatment. Melbourne: International Diabetes Institute; 2000. p. 11-2.

17. Craig CL, Marshall AL, Sjostrom M, Bauman AE, Booth ML, Ainsworth BE, Pratt $M$, Ekelund U, Yngve A, Sallis JF, et al. International physical activity questionnaire: 12-country reliability and validity. Med Sci Sports Exerc. 2003; 35(8):1381-95.

18. Quan H, Sundararajan V, Halfon P, Fong A, Burnand B, Luthi JC, Saunders LD, Beck CA, Feasby TE, Ghali WA. Coding algorithms for defining comorbidities in ICD-9-CM and ICD-10 administrative data. Med Care. 2005; 43(11):1130-9.

19. Honda T, Pun VC, Manjourides J, Suh H. Associations between long-term exposure to air pollution, glycosylated hemoglobin and diabetes. Int J Hyg Environ Health. 2017;220(7):1124-32.

20. Yang B-Y, Qian Z, Li S, Chen G, Bloom MS, Elliott M, Syberg KW, Heinrich J, Markevych I, Wang S-Q, et al. Ambient air pollution in relation to diabetes and glucose-homoeostasis markers in China: a cross-sectional study with findings from the 33 communities Chinese health study. Lancet Planetary Health. 2018;2(2):e64-73.

21. Yang B-Y, Bloom MS, Markevych I, Qian ZM, Vaughn MG, CummingsVaughn LA, Li S, Chen G, Bowatte G, Perret JL. Exposure to ambient air pollution and blood lipids in adults: the 33 communities Chinese health study. Environ Int. 2018;119:485-92.
22. Yitshak Sade M, Kloog I, Liberty IF, Schwartz J, Novack V. The association between air pollution exposure and glucose and lipids levels. J Clin Endocrinol Metab. 2016;101(6):2460-7.

23. Jerrett M, McConnell R, Wolch J, Chang R, Lam C, Dunton G, Gilliland F, Lurmann F, Islam T, Berhane K. Traffic-related air pollution and obesity formation in children: a longitudinal, multilevel analysis. Environ Health. 2014;13(1):49.

24. Li W, Dorans KS, Wilker EH, Rice MB, Schwartz J, Coull BA, Koutrakis P, Gold DR, Fox CS, Mittleman MA. Residential proximity to major roadways, fine particulate matter, and adiposity: the Framingham heart study. Obesity. 2016;24(12):2593-9.

25. Alderete TL, Habre R, Toledo-Corral CM, Berhane K, Chen Z, Lurmann FW, Weigensberg MJ, Goran MI, Gilliland FD. Longitudinal associations between ambient air pollution with insulin sensitivity, $\beta$-cell function, and adiposity in Los Angeles Latino children. Diabetes. 2017;66(7):1789-96.

26. Li W, Wilker EH, Dorans KS, Rice MB, Schwartz J, Coull BA, Koutrakis P, Gold DR, Keaney JF Jr, Lin H, et al. Short-Term Exposure to Air Pollution and Biomarkers of Oxidative Stress: The Framingham Heart Study. J Am Heart Assoc. 2016;5(5):e002742.

27. Sun Q, Wang A, Jin X, Natanzon A, Duquaine D, Brook RD, Aguinaldo J-GS, Fayad ZA, Fuster $V$, Lippmann $M$, et al. Long-term air pollution exposure and acceleration of atherosclerosis and vascular inflammation in an animal model. JAMA. 2005;294(23):3003-10.

28. Rajagopalan S, Al-Kindi SG, Brook RD. Air pollution and cardiovascular disease: JACC state-of-the-art review. J Am Coll Cardiol. 2018;72(17):2054-70.

29. Li R, Navab M, Pakbin P, Ning Z, Navab K, Hough G, Morgan TE, Finch CE, Araujo JA, Fogelman AM, et al. Ambient ultrafine particles alter lipid metabolism and HDL anti-oxidant capacity in LDLR-null mice. J Lipid Res. 2013;54(6):1608-15.

30. Li J, Zhou C, Xu H, Brook RD, Liu S, Yi T, Wang Y, Feng B, Zhao M, Wang X, et al. Ambient air pollution is associated with $\mathrm{HDL}$ (high-density lipoprotein) dysfunction in healthy adults. Arterioscler Thromb Vasc Biol. 2019;39(3):513-22.

31. Shanley RP, Hayes RB, Cromar KR, Ito K, Gordon T, Ahn J. Particulate air pollution and clinical cardiovascular disease risk factors. Epidemiology. 2016; 27(2):291-8.

32. Hansen AB, Ravnskjaer L, Loft S, Andersen KK, Brauner EV, Baastrup R, Yao C, Ketzel $M$, Becker $T$, Brandt J, et al. Long-term exposure to fine particulate matter and incidence of diabetes in the Danish nurse cohort. Environ Int. 2016;91:243-50

33. Ryou HG, Heo J, Kim SY. Source apportionment of PM10 and PM2.5 air pollution, and possible impacts of study characteristics in South Korea. Environ Pollut (Barking, Essex : 1987). 2018;240:963-72.

34. Miller FJ, Gardner DE, Graham JA, Lee RE, Wilson WE, Bachmann JD. Size considerations for establishing a standard for inhalable particles. J Air Pollut Control Assoc. 1979:29(6):610-5.

35. Kan H, London SJ, Chen G, Zhang Y, Song G, Zhao N, Jiang L, Chen B. Differentiating the effects of fine and coarse particles on daily mortality in Shanghai, China. Environ Int. 2007:33(3):376-84.

36. Samoli E, Stafoggia M, Rodopoulou S, Ostro B, Declercq C, Alessandrini E, Diaz J, Karanasiou A, Kelessis AG, Le Tertre A, et al. Associations between fine and coarse particles and mortality in Mediterranean cities: results from the MED-PARTICLES project. Environ Health Perspect. 2013;121(8):932-8.

37. Rao X, Patel P, Puett $R$, Rajagopalan $S$. Air pollution as a risk factor for type 2 diabetes. Toxicol Sci. 2014;143(2):231-41.

38. Lee IM, Shiroma EJ, Lobelo F, Puska P, Blair SN, Katzmarzyk PT. Lancet Physical Activity Series Working G: Effect of physical inactivity on major noncommunicable diseases worldwide: an analysis of burden of disease and life expectancy. Lancet (London, England). 2012;380(9838):219-29.

39. Bruunsgaard $\mathrm{H}$. Physical activity and modulation of systemic low-level inflammation. J Leukoc Biol. 2005;78(4):819-35.

40. Brook RD, Xu X, Bard RL, Dvonch JT, Morishita M, Kaciroti N, Sun Q, Harkema J, Rajagopalan S. Reduced metabolic insulin sensitivity following sub-acute exposures to low levels of ambient fine particulate matter air pollution. Sci Total Environ. 2013:448:66-71.

41. Geiser M, Kreyling WG. Deposition and biokinetics of inhaled nanoparticles. Particle and Fibre Toxicology. 2010;7(1):2.

42. Thompson PD, Franklin BA, Balady GJ, Blair SN, Corrado D, Estes NAM 3rd, Fulton JE, Gordon NF, Haskell WL, Link MS, et al. Exercise and acute cardiovascular events placing the risks into perspective: a scientific statement from the American Heart Association Council on nutrition, 
physical activity, and metabolism and the council on clinical cardiology. Circulation. 2007;115(17):2358-68.

43. World Health Organization Regional Office for Europe. Health effects of particulate matter. Copenhagen: World Health Organization Regional Office for Europe; 2013.

44. Ostro B, Broadwin R, Green S, Feng WY, Lipsett M. Fine particulate air pollution and mortality in nine California counties: results from CALFINE. Environ Health Perspect. 2006;114(1):29-33.

45. Qiu H, Yu IT, Tian L, Wang X, Tse LA, Tam W, Wong TW. Effects of coarse particulate matter on emergency hospital admissions for respiratory diseases: a time-series analysis in Hong Kong. Environ Health Perspect. 2012; 120(4):572-6.

\section{Publisher's Note}

Springer Nature remains neutral with regard to jurisdictional claims in published maps and institutional affiliations.

- fast, convenient online submission

- thorough peer review by experienced researchers in your field

- rapid publication on acceptance

- support for research data, including large and complex data types

- gold Open Access which fosters wider collaboration and increased citations

- maximum visibility for your research: over $100 \mathrm{M}$ website views per year

At $\mathrm{BMC}$, research is always in progress. 